\title{
CS Research Square

\section{Sustained mitochondrial damages in cardiomyocytes in a patient with severe propofol infusion syndrome (PRIS)}

\section{Satoshi Karasawa}

Chiba University Graduate School of Medicine

Taka-aki Nakada ( $\square$ taka.nakada@nifty.com )

Chiba University Graduate School of Medicine https://orcid.org/0000-0002-4480-556X

Naoto Mori

Chiba University Graduate School of Medicine

Michiko Daimon

Chiba University Graduate School of Medicine

Hideyuki Miyauchi

Chiba University Graduate School of Medicine

\section{Tetsuya Kanai}

Chiba University Graduate School of Medicine

Hiroyuki Takano

Chiba University Graduate School of Medicine

Yoshio Kobayashi

Chiba University Graduate School of Medicine

\section{Shigeto Oda}

Chiba University Graduate School of Medicine

\section{Case Report}

Keywords: Mitochondria, arrhythmia, cardiac failure

Posted Date: December 27th, 2018

DOI: https://doi.org/10.21203/rs.2.150/v1

License: (c) (i) This work is licensed under a Creative Commons Attribution 4.0 International License. Read Full License 


\section{Abstract}

Background: Propofol infusion syndrome (PRIS) is rare but possibly lethal. Mitochondrial damage is suggested as a potential pathogenesis. However, investigations on the mitochondrial damages in cardiomyocytes in patients with PRIS are insufficient.

Case presentation: A 20-year-old man with Guillain-Barré syndrome had propofol administration for sedation. On day 13 , he had hypotension with ST elevation in electrocardiogram, acute kidney injury, hyperkalemia and severe rhabdomyolysis. He was transferred to our ICU on suspicion of PRIS.

Administration of noradrenaline and hemodialysis were initiated, and fasciotomy was required for PRISrhabdomyolysis. He gradually recovered and was discharged from the ICU on day 30. On day 37, he repeatedly presented sinus bradycardia and conduction disturbance eventually requiring temporary pacing. Cardiac 18F-fluorodeoxyglucose positron emission tomography (18F-FDG PET) on day 67 demonstrated heterogeneous 18F-FDG uptake in left ventricle. Electron microscopic investigation of the endomyocardial biopsy on day 75 revealed abnormal findings in the mitochondria of the cardiomyocytes including myelinization of the cristae. He was discharged with normal cardiac function without arrhythmia on day 192.

Conclusions: This is a case of PRIS with evidence of mitochondrial damage in both morphological and functional aspects. Sustained mitochondrial damage may be a therapeutic target beyond the initial therapy of discontinuing propofol administration.

Keywords: Mitochondria, arrhythmia, cardiac failure

\section{Background}

Propofol is extensively used in the intensive care units (ICU) for sedation. Propofol infusion syndrome (PRIS) is rare but a potentially lethal adverse event [1]. Here we report a severe case of PRIS.

\section{Case Presentation}

A 20-year-old man, who previously had been healthy, was admitted for the treatment of Guillain-Barré syndrome. On day 6 , he required mechanical ventilation due to progressive muscle weakness; propofol ( $3.5 \mathrm{mg} / \mathrm{kg} / \mathrm{hour}$ ) was administered for 5 days for sedation. On day 13 , he had hypotension with abnormal electrocardiogram findings (ST elevation in II, III, and $\mathrm{aV}_{\mathrm{F}}$ ), acute kidney injury, hyperkalemia and severe rhabdomyolysis (serum creatinine phosphokinase, 271,700 IU/L). He was transferred to our ICU on suspicion of PRIS. Administration of noradrenaline $(0.3 \mathrm{microgram} / \mathrm{kg} / \mathrm{min})$ and hemodialysis were initiated, and fasciotomy was required for compartment syndrome of lower legs due to PRISrhabdomyolysis. He gradually recovered from cardiac and renal dysfunction and was discharged from the ICU on day 30 . On day 37 , he repeatedly presented sinus bradycardia and right bundle branch block eventually requiring temporary pacing, with finding of pericardial effusion on echocardiography. Detailed examination including cardiac ${ }^{18} \mathrm{~F}$-fluorodeoxyglucose positron emission tomography ( ${ }^{18} \mathrm{~F}$-FDG PET) was 
conducted to evaluate whether these late phase cardiac events were related to PRIS. Cardiac ${ }^{18} \mathrm{~F}-\mathrm{FDG}$ PET on day 67 demonstrated heterogeneous ${ }^{18}$ F-FDG uptake in left ventricle (Figure 1). Electron microscopic investigation of the endomyocardial biopsy on day 75 revealed abnormal findings in the mitochondria of the cardiomyocytes including myelinization of the cristae (Figure 2). He was discharged with normal cardiac function without arrhythmia on day 192.

\section{Discussion And Conclusions}

Mitochondrial damage is suggested as a potential pathogenesis of PRIS [2, 3]. Mitochondrial damage was observed as a morphological finding in an electron microscopic evaluation of the heart in an autopsy case of PRIS [4]. Similarly, mitochondrial damage was observed in the endomyocardial biopsy two months after the onset in the present case. Mitochondrial damage can also be detected as a functional impairment of fatty acid utilization with alternatively increased glucose utilization [5]. The uptake of a glucose analog ( ${ }^{18} \mathrm{~F}-\mathrm{FDG}$ ) in left ventricle on day 67 (Figure 1) in the present case implies a shift in the energy substrate of cardiomyocytes from fatty acid to glucose, suggesting mitochondrial damage. To the best of our knowledge, this is the first to report a case of PRIS with evidence of mitochondrial damage in both morphological and functional aspects. Sustained mitochondrial damage may be a therapeutic target beyond the initial therapy of discontinuing propofol administration.

\section{Abbreviations}

ICU=intensive care units

PRIS=Propofol infusion syndrome

${ }^{18} \mathrm{~F}$-FDG PET $={ }^{18} \mathrm{~F}$-fluorodeoxyglucose positron emission tomography

\section{Declarations}

\section{Ethics approval and consent to participate}

Not applicable for the case report

\section{Consent for publication}

Written informed consent for the publication of individual details was obtained from the patient whose case is reported here.

\section{Availability of data and material}


The images in the report are available from the corresponding author on reasonable request.

\section{Competing interests}

We declare that we have no competing interests.

\section{Funding}

None

\section{Authors' contributions:}

SK and TN: These authors contributed to drafting and critical revision of the manuscript for important intellectual content. NM, MD, HM, TK, HT, YK and SO: These authors contributed to critical revision of the manuscript for important intellectual content. All authors read and approved the final manuscript.

\section{Acknowledgements}

Not Applicable

\section{References}

1. Vanlander AV, Okun JG, de Jaeger A, Smet J, De Latter E, De Paepe B, Dacremont G, Wuyts B, Vanheel $B$, De Paepe $P$ et al: Possible pathogenic mechanism of propofol infusion syndrome involves coenzyme q. Anesthesiology 2015;122:343-352.

2. Krajcova A, Waldauf $P$, Andel M, Duska F: Propofol infusion syndrome: a structured review of experimental studies and 153 published case reports. Crit Care 2015;19:398.

3. Wolf A, Weir P, Segar P, Stone J, Shield J: Impaired fatty acid oxidation in propofol infusion syndrome. Lancet 2001;357:606-607.

4. Vollmer JP, Haen S, Wolburg H, Lehmann R, Steiner J, Reddersen S, Fend F, Fallier-Becker P: Propofol Related Infusion Syndrome: Ultrastructural Evidence for a Mitochondrial Disorder. Crit Care Med 2018;46:e91-e94.

5. Vandanmagsar B, Warfel JD, Wicks SE, Ghosh S, Salbaum JM, Burk D, Dubuisson OS, Mendoza TM, Zhang J, Noland RC et al: Impaired Mitochondrial Fat Oxidation Induces FGF21 in Muscle. Cell Rep 2016;15:1686-1699. 


\section{Figures}

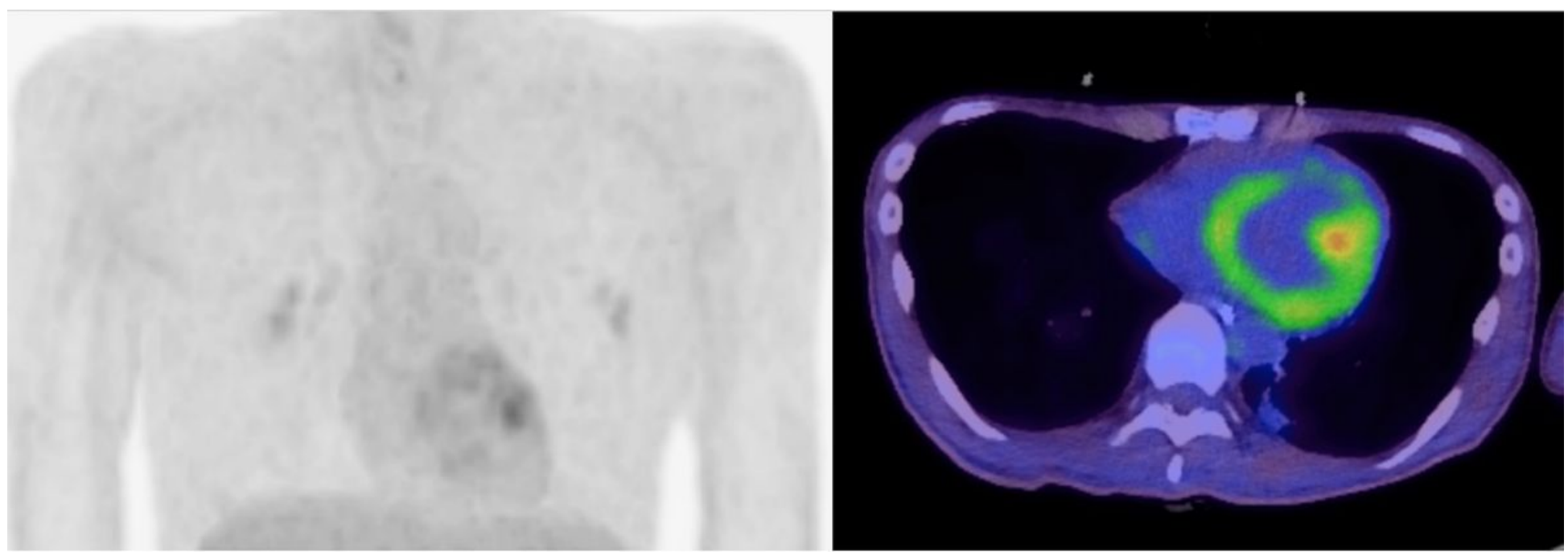

\section{Figure 1}

18F-fluorodeoxyglucose positron emission tomography $18 \mathrm{~F}$-fluorodeoxyglucose positron emission tomography showed heterogeneous $18 \mathrm{~F}-\mathrm{FDG}$ uptake in left ventricle. 


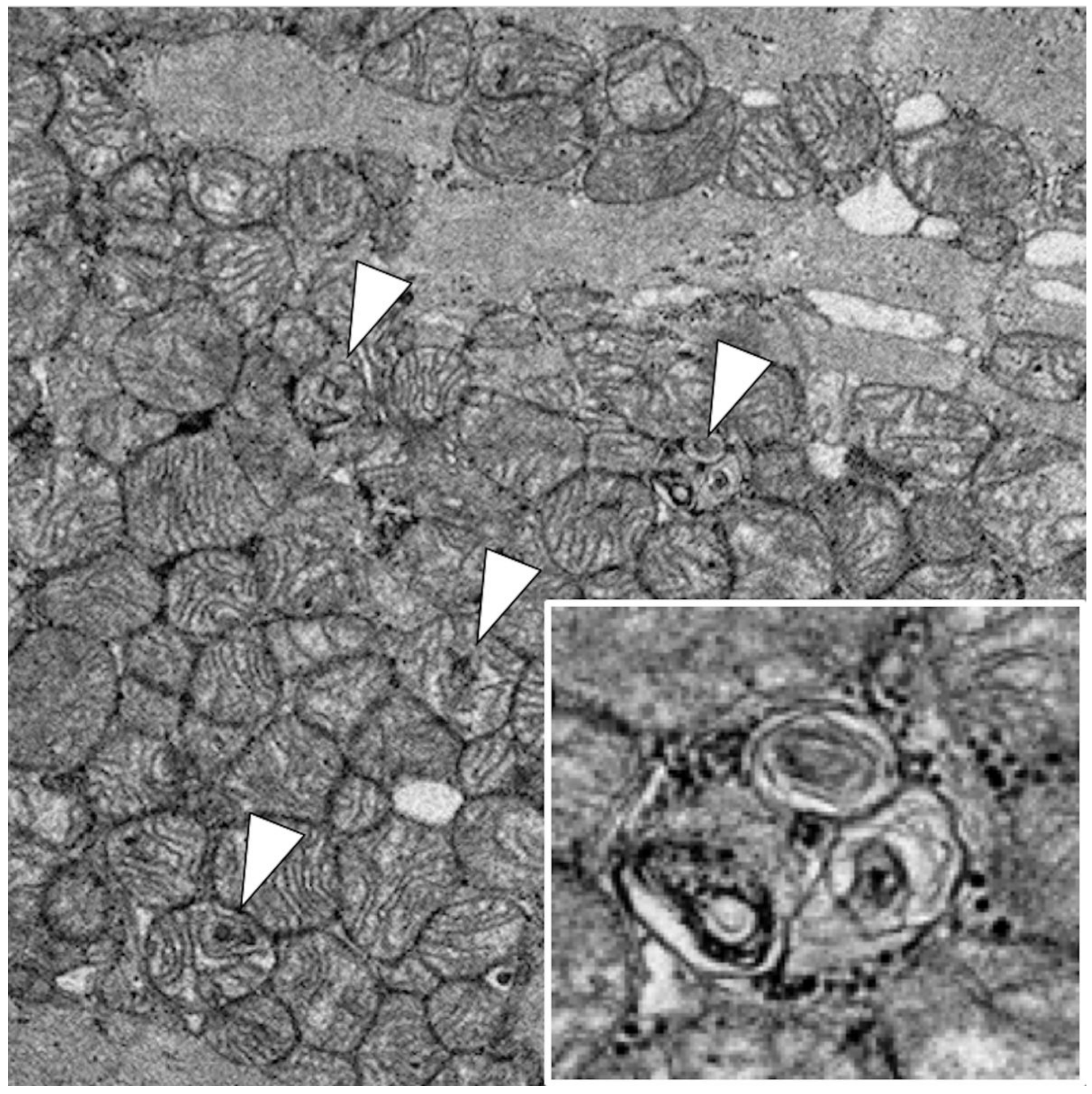

\section{Figure 2}

Electron microscopic investigation of endomyocardial biopsy Electron microscopy revealed mitochondrial myelinizations of the cristae in cardiomyocyte. Arrows indicate cardiomyocytes with the mitochondrial injury.

\section{Supplementary Files}


This is a list of supplementary files associated with this preprint. Click to download.

- supplement1.pdf 